\title{
NORMAS DRIS PARA A CULTURA DO COQUEIRO HÍBRIDO NO ESTADO DO PARÁ ${ }^{1}$
}

\author{
EDUARDO CÉZAR MEDEIROS SALDANHA ${ }^{2 *}$, MÁRIO LOPES DA SILVA JÚNIOR ${ }^{2}$, \\ RICARDO SHIGUERU OKUMURA ${ }^{2}$, PAULO GUILHERME SALVADOR WADT ${ }^{3}$
}

\begin{abstract}
RESUMO - O Sistema Integrado de Diagnose e Recomendação (DRIS) é baseado no cálculo de um índice para cada nutriente. Para o cálculo das normas DRIS há a necessidade de um banco de dados contendo resultados de análise foliar e produtividade. No Estado do Pará não existem normas DRIS desenvolvidas para a cultura do coqueiro híbrido. O objetivo do trabalho foi desenvolver essas normas para a cultura do coqueiro híbrido. $\mathrm{O}$ trabalho foi realizado em área comercial de coqueiro no município de Moju (PA). O banco de dados foi formado por 134 amostras no período de 2001 a 2011. Foram obtidas a média, o desvio padrão, o coeficiente de variação e a variância das relações das concentrações dos nutrientes, além dos coeficientes de correlação entre a relação de cada par de nutrientes e a produtividade de frutos. As normas foram estabelecidas com base na população de alta produtividade. Das 110 relações 55 foram selecionadas para compor as referidas normas, utilizando dois critérios de seleção das relações entre os nutrientes. Os maiores valores de desvio padrão, variância e coeficiente de variação foram apresentados para os teores foliares dos micronutrientes, quais sejam: ferro, manganês e boro. Os nutrientes que apresentaram maiores percentagens de amostras com teores abaixo dos níveis adequados adotados foram o $\mathrm{Mg}$ e $\mathrm{Ca}$, enquanto que as amostras com teores foliares acima ou iguais aos níveis adequados foram o $\mathrm{Fe}, \mathrm{Mn}$ e $\mathrm{Ca}$.
\end{abstract}

Palavras-chave: Cocus nucifera L.. Diagnose nutricional. Padrões de referência.

\section{ESTABLISHMENT OF DRIS NORMS FOR GROWING HYBRID COCONUT IN PARÁ}

\begin{abstract}
The Integrated Diagnosis and Recommendation (DRIS) is based on the calculation of an index for each nutrient. For the calculation of DRIS norms, there is a need a database containing the results of leaf analysis and yield. In Pará State, no DRIS norms developed for the cultivation of hybrid coconut. The objective was to develop DRIS norms for this culture. The aim was carried at the Socôco farm in Moju PA. The database consisted of 134 observations for the period 2001-2011. We obtained the mean, standard deviation, variance and coefficient of variation of the relationships of concentrations of nutrients, and the coefficients of correlation between the ratio of each pair of nutrients and fruit yield. DRIS norms were established based on the population of high productivity. Of the 110 relationships, 55 were selected to DRIS norms, using two selection criteria of relationships between nutrients. The highest values of standard deviation, variance and coefficient of variation were presented to the leaf levels of the micronutrients iron, manganese, and boron. While the nutrients showed higher percentages of samples with levels below the adequate levels used were $\mathrm{Mg}$ and $\mathrm{Ca}$, and finally the nutrients in leaf samples presented above or equal to the adequate levels contents were $\mathrm{Fe}, \mathrm{Mn}$ and $\mathrm{Ca}$.
\end{abstract}

Keywords: Cocus nucifera L.. Nutritional diagnosis. Reference standards.

\footnotetext{
*Autor para correspondência

${ }^{1}$ Recebido para publicação em 10/11/2014; aceito em 15/06/2015.

Trabalho de Tese de Doutorado em Agronomia do primeiro autor.

${ }^{2}$ Universidade Federal Rural da Amazônia - UFRA, CEP 66077-830, Belém (PA); ecmsaldanha@yahoo.com.br, mario.silva@ufra.edu.br, ricardo.okumura@ufra.edu.br.

${ }^{3}$ Empresa Brasileira de Pesquisa Agropecuária, Centro de Pesquisa Agroflorestal de Rondônia, CEP 78815-800, Porto Velho (RO); paulogswadt@dris.com.br.
} 


\section{INTRODUÇ̃̃̃O}

A cultura do coqueiro é nutricionalmente exigente por demandar elevada quantidade de nutrientes, uma vez que a planta se desenvolve de forma contínua, com ocorrência simultânea da floração, frutificação e maturação dos frutos (SOBRAL; NOGUEIRA, 2008). Teixeira et al. (2005) apontaram que o acompanhamento nutricional da cultura do coqueiro, por meio de análises de tecido foliar, seja feito como técnica auxiliar para a recomendação de fertilizantes.

Entre os métodos de interpretação dos resultados das análises químicas foliares, o Sistema Integrado de Diagnose e Recomendação (DRIS) tem lugar de destaque. A interpretação foliar por este método é baseado em um índice gerado para cada nutriente, o qual permite determinar a disponibilidade relativa de cada nutriente. Esse índice é obtido comparando-se as relações na forma de quociente entre um nutriente e cada um dos demais presentes na amostra sob diagnose, comparando esse valor com valores de referência envolvendo as mesmas relações em populações sadias e produtivas, denominada de população de referência (CASTAMANN et al., 2012).

A primeira etapa no estabelecimento do método DRIS está no desenvolvimento das normas ou padrões de referência. Na região Amazônica se destacam os trabalhos de Dias et al. (2010) e Wadt e Dias (2012), os quais desenvolveram normas DRIS para as culturas do cupuaçu e café Conilon, respectivamente. As normas são as estatísticas, com média e variância, das relações entre os teores dos nutrientes, representando culturas em boas condições nutricionais (BEAUFILS, 1973). A relação entre um par de nutrientes pode ser tanto direta como inversa como, por exemplos, N/P e P/N, respectivamente. Contudo, nos cálculos do método DRIS, alguns autores utilizam apenas um tipo de expressão para relacionar cada par de nutrientes (NACHTIGALL; DECHEN, 2007).

Vários critérios para selecionar a expressão mais adequada (direta ou inversa) têm sido propostos. O mais utilizado é o critério da maior relação de variâncias entre as populações de baixa e alta produtividades (LETZSCH, 1985) chamado de "valor F". Nachtigall e Dechen (2007), trabalhando com a cultura da macieira, descreveram o critério denominado valor "R", que compreende o cálculo dos coeficientes de correlação (R) entre os valores da produtividade e a razão entre os pares de nutrientes, tanto na ordem direta como na ordem inversa. Por esse critério escolhe-se a ordem da razão que resulta no mais alto valor absoluto do coeficiente de correlação.

Na cultura do coqueiro, os resultados das análises foliares são tradicionalmente interpretados com a utilização do critério do nível crítico e das faixas de suficiência, sendo os primeiros valores de níveis críticos foliares obtidos na Costa do Marfim, comparando os resultados de experimentos de campo e baseando-se na relação entre as doses de nutrientes fornecidas aos solos, resposta das plantas pelas colheitas e composição mineral da cultura (MANCIOT et al., 1980), havendo, no entanto, grande desvantagem e fragilidade de assertividade, pois os valores de referência foram estabelecidos com resultados de pesquisas realizadas, principalmente entre os anos de 1970 e 1980 e com cultivares de diferentes potenciais produtivos, o que pode gerar diagnósticos nutricionais equivocados.

Nas condições Amazônicas, especificamente na região nordeste do Estado do Pará, o que se observa é a ausência de normas DRIS regionais para a cultura coqueiro variedade híbrido. Assim, objetivou -se com o trabalho desenvolver as normas DRIS em função de diferentes critérios de seleção das relações entre os nutrientes para a cultura do coqueiro híbrido no Estado do Pará.

\section{MATERIAL E MÉTODOS}

O trabalho foi realizado em área de produção comercial de coqueiro localizada no município de Moju, no Estado do Pará (“02 $02^{\circ}$ '00” S e $48^{\circ}$ 22'30" W), em que predominam solos pertencentes a classe dos Latossolos Amarelos (EMBRAPA, 2013). O clima da região é do tipo Ami, segundo a classificação de Köppen, caracterizando-se como clima tropical chuvoso, sem variação térmica estacional e apresentando anualmente um total pluviométrico médio de $2500 \mathrm{~mm}$.

Utilizou-se banco de dados contendo resultados de análises foliares e de produtividade agrícola do coqueiro referentes ao período de 2001 a 2011 . Foram selecionadas 134 unidades de produção para compor o banco de dados. Em cada unidade selecionada foram coletadas informações referentes ao histórico da área, material genético, ano de plantio, idade dos plantios na data de coleta e espaçamento. Foram coletadas amostras compostas de tecido foliar e registrado a produtividade média. Cada amostra composta foi formada a partir de material coletado de 25 plantas por área, sendo realizadas no período de 15 de dezembro a 15 de janeiro em todos os anos da avaliação, considerado período indicado para a coleta de folhas na cultura do coqueiro na região Amazônica (LINS; VIÉGAS, 2008).

Fora coletada a folha de posição número 14 (SOBRAL, 1998). Em cada folha coletaram-se seis folíolos intactos da parte central do ráquis, sendo três de cada lado. Em cada folíolo aproveitou-se os 10 $\mathrm{cm}$ centrais, eliminando-se as bordas e a nervura central. Os folíolos foram limpos com algodão embebido em água destilada e depois colocadas em sacos de papel etiquetados, contendo as informações de cada amostra. As amostras foram colocadas para secar em estufa de circulação forçada de ar à temperatura de $70^{\circ} \mathrm{C}$ até massa constante. Após a secagem do material foram enviadas para o Laboratório de 
Análise de tecido Vegetal da Esalq/USP para determinação dos teores de $\mathrm{N}, \mathrm{P}, \mathrm{K}, \mathrm{Ca}, \mathrm{Mg}, \mathrm{B}, \mathrm{Cu}, \mathrm{Fe}$, Mn e Zn, de acordo com Malavolta et al. (1997).

$\mathrm{O}$ banco de dados foi dividido em dois grupos ou populações (PAP = População de Alta Produtividade e PBP = População de Baixa Produtividade) em função da produtividade de frutos medida em número de frutos planta ${ }^{-1}$ ano $^{-1}$. Optou-se por definir como área de alta produtividade aquelas que apresentaram produtividade superior a 130 frutos planta ${ }^{-1} \mathrm{ano}^{-1}$, conforme padrões de produtividade estabelecidos pela literatura (LINS et al., 2003, MOHANDAS, 2012).

Foram obtidas a média, o desvio padrão, o coeficiente de variação e a variância das relações das concentrações dos nutrientes, dois a dois, das amostras de folhas das safras 2001 a 2011, além dos coeficientes de correlação entre a relação de cada par de nutrientes e a produtividade de frutos. Para a escolha da ordem da razão dos nutrientes foram avaliados dois critérios. O primeiro critério, descrito por Letzsch (1985), denominado de "valor F", o qual consiste no cálculo da razão de variância das relações entre nutrientes entre o grupo de referência (r) e de baixa produtividade (b), tanto na ordem direta como inversa. É selecionada a ordem da relação que apresentar maior razão de variância entre o grupo de alta e o de baixa produtividade:

Se: $\left[s^{2}(A / B)_{b} / s^{2}(A / B)_{b}\right]>\left[s^{2}(B / A)_{b} / s^{2}(B / A)_{b}\right]$ então: relação na norma $=A / B$ Se: $\left[s^{2}(A / B)_{b} / s^{2}(A / B)_{b}\right]<\left[s^{2}(B / A)_{b} / s^{2}(B / A)_{b}\right]$ então: relação na norma $=B / A$

Em que: $s^{2}(A / B)_{b}=$ Variância da razão entre as concentrações dos nutrientes $\mathrm{A}$ e $\mathrm{B}$ da população de baixa produtividade; $s^{2}(A / B)_{r}=$ Variância da razão entre as concentrações dos nutrientes $\mathrm{A}$ e $\mathrm{B}$ da população de referência; $\mathrm{s}^{2}(\mathrm{~B} / \mathrm{A})_{\mathrm{b}}=$ Variância da razão entre as concentrações dos nutrientes $\mathrm{B}$ e $\mathrm{A}$ da população de baixa produtividade; e $\mathrm{s}^{2}(\mathrm{~B} / \mathrm{A})_{\mathrm{r}}=$ Vari- ância da razão entre as concentrações dos nutrientes B e A da população de referência.

O segundo critério, descrito por Nachtigall e Dechen (2007), denominado de "Valor R", consiste no cálculo dos coeficientes de correlação (r) entre os valores de produtividade (frutos planta ${ }^{-1}$ ano $^{-1}$ ) e a relação entre os pares de nutrientes, tanto na ordem direta como na ordem inversa. É selecionada a ordem da relação que apresentar maior valor absoluto do coeficiente de correlação $(\mathrm{r})$ :

Se: $\left[r_{A / B}\right]>\left[r_{B / A}\right]$ então: relação na norma $=\mathrm{A} / \mathrm{B}$

Se: $\left[r_{A / B}\right]<\left[r_{B} / A\right]$ então: relação na norma $=\mathrm{B} / \mathrm{A}$

Em que: $\left|\mathrm{r}_{\mathrm{A} / \mathrm{B}}\right|=$ Valor absoluto do coeficiente de correlação entre a produtividade de frutos e a razão entre as concentrações dos nutrientes A e B na população de referência; e $\left|\mathrm{r}_{\mathrm{B} / \mathrm{A}}\right|=$ Valor absoluto do coeficiente de correlação entre a produtividade de frutos e a razão entre as concentrações dos nutrientes B e A na população de referência.

Foram calculadas todas as relações possíveis entre os nutrientes dois a dois (em sua forma direta $\mathrm{e}$ inversa) com suas respectivas médias, desvio padrão, coeficiente de variação e variância das amostras de folhas das safras 2001 a 2011, além dos coeficientes de correlação entre a relação de cada par de nutrientes e a produtividade de frutos. Os cálculos foram realizados para as populações de baixa produtividade e de alta produtividade. No entanto, as normas foram obtidas a partir das relações na população de alta produtividade. Os resultados de concentrações de nutrientes nas folhas foram submetidos a análise estatística descritiva utilizando a ferramenta de análise de dados em planilha eletrônica Microsoft Excel e comparados com os níveis críticos foliares definidos como padrão para a cultura (Tabela 1) utilizando o software estatístico Assistat ${ }^{\circledR}$ (SILVA; AZEVEDO, 2009).

Tabela 1. Níveis críticos foliares para coqueiro híbrido, na folha de posição 14, de acordo com diferentes autores para interpretação de análise foliar.

\begin{tabular}{lcc}
\hline Nutriente & Silva e Faria 2001 & Lins e Viégas $2008^{*}$ \\
\hline $\mathrm{N}\left(\mathrm{g} \mathrm{kg}^{-1}\right)$ & 22 & 22 \\
$\mathrm{P}\left(\mathrm{g} \mathrm{kg}^{-1}\right)$ & 1,2 & 1,4 \\
$\mathrm{~K}\left(\mathrm{~g} \mathrm{~kg}^{-1}\right)$ & 14 & 14 \\
$\mathrm{Ca}\left(\mathrm{g} \mathrm{kg}^{-1}\right)$ & 5,0 & 3,0 \\
$\mathrm{Mg}\left(\mathrm{g} \mathrm{kg}^{-1}\right)$ & 2,0 & 2,2 \\
$\mathrm{~S}\left(\mathrm{~g} \mathrm{~kg}^{-1}\right)$ & 2,5 & 1,5 \\
$\mathrm{~B}\left(\mathrm{mg} \mathrm{kg}^{-1}\right)$ & 10 & 20 \\
$\mathrm{Cu}\left(\mathrm{mg} \mathrm{kg}^{-1}\right)$ & 4,0 & 10 \\
$\mathrm{Fe}\left(\mathrm{mg} \mathrm{kg}^{-1}\right)$ & 40 & 40 \\
$\mathrm{Mn}\left(\mathrm{mg} \mathrm{kg}^{-1}\right)$ & 100 & 70 \\
$\mathrm{Zn}\left(\mathrm{mg} \mathrm{kg}^{-1}\right)$ & 15 & 8 \\
\hline
\end{tabular}

*Níveis críticos foliares adotados regionalmente 
As normas DRIS para a cultura do coqueiro híbrido foram estabelecidas com base nas relações entre os nutrientes na população de alta produtividade (PAP). Das 110 relações entre os nutrientes obtidas 55 foram selecionadas para compor as normas DRIS para a cultura do coqueiro híbrido em cada conjunto de normas geradas.

\section{RESULTADOS E DISCUSSÃO}

Os maiores valores de desvio padrão, variância e coeficiente de variação foram encontrados nos teores foliares de ferro, manganês e boro (Tabela 2). Dias et al. (2013), em trabalho desenvolvendo nor- mas DRIS multivariadas para o citros no Estado do Amazonas com uma população de 120 áreas comerciais avaliadas, também constataram que os maiores coeficientes de variação foram encontrados para os micronutrientes ferro e cobre $(128,1$ e $114,9 \%)$, respectivamente. Para os demais nutrientes, a variabilidade verificada nos teores foliares pode ser considerada adequada, conforme constatação de Wairegi e Van Asten (2012), apresentando valores de desvio padrão, variância, coeficientes de variação, curtose e assimetria aceitáveis para o tipo de estudo proposto. Essa alta variabilidade pode estar relacionada, possivelmente, a variações ocorridas nas determinações laboratoriais desses nutrientes.

Tabela 2. Estatística descritiva da produtividade do coqueiro $\left(\right.$ coco planta $^{-1}$ ano $\left.^{-1}\right)$, teores foliares de nitrogênio (N), fósforo $(\mathrm{P})$, potássio $(\mathrm{K})$, cálcio $(\mathrm{Ca})$, magnésio $(\mathrm{Mg})$, enxofre $(\mathrm{S})$, boro $(\mathrm{B})$, cobre $(\mathrm{Cu})$, ferro $(\mathrm{Fe})$, manganês $(\mathrm{Mn})$ e zinco $(\mathrm{Zn})$. Valores médios (Md), medianos (Med), mínimo (Min), máximo (Max), desvio padrão (s), coeficiente de variação (CV), variância $\left(\mathrm{s}^{2}\right)$, coeficiente de curtose (Curt) e coeficiente de assimetria (Assim) de 134 talhões de área comercial de coqueiro no município de Moju (PA) no período de 2001 a 2011.

\begin{tabular}{lccccccccc}
\hline Variável & Md & Med & Min & Max & s & CV & $\mathrm{s}^{2}$ & Curt & Assim \\
\hline coco planta $^{-1} \mathrm{ano}^{-1}$ & 109 & 113 & 10 & 148 & 29,3 & 26,8 & 855,40 & 1,85 & $-1,39$ \\
$\mathrm{~N}\left(\mathrm{~g} \mathrm{~kg}^{-1}\right)$ & 20,2 & 19,9 & 3,4 & 30,1 & 3,1 & 15,3 & 9,50 & 7,62 & $-1,12$ \\
$\mathrm{P}\left(\mathrm{g} \mathrm{kg}^{-1}\right)$ & 1,4 & 1,4 & 0,9 & 2,0 & 0,2 & 12,8 & 0,03 & 1,02 & 0,91 \\
$\mathrm{~K}\left(\mathrm{~g} \mathrm{~kg}^{-1}\right)$ & 14,1 & 13,9 & 9,4 & 25 & 2,4 & 16,8 & 5,66 & 2,20 & 0,83 \\
$\mathrm{Ca}\left(\mathrm{g} \mathrm{kg}^{-1}\right)$ & 3,6 & 3,5 & 2,1 & 6,6 & 0,9 & 25,1 & 0,81 & $-0,17$ & 0,53 \\
$\mathrm{Mg}\left(\mathrm{g} \mathrm{kg}^{-1}\right)$ & 1,5 & 1,4 & 0,9 & 2,1 & 0,2 & 16,5 & 0,06 & $-0,51$ & 0,21 \\
$\mathrm{~S}\left(\mathrm{~g} \mathrm{~kg}^{-1}\right)$ & 1,3 & 1,2 & 0,6 & 2,5 & 0,3 & 24,4 & 0,09 & 0,67 & 0,58 \\
$\mathrm{~B}\left(\mathrm{mg} \mathrm{kg}^{-1}\right)$ & 15,7 & 15,1 & 5,0 & 36,4 & 5,1 & 32,5 & 26,11 & 2,31 & 0,95 \\
$\mathrm{Cu}\left(\mathrm{mg} \mathrm{kg}^{-1}\right)$ & 4,2 & 4,1 & 2,0 & 8,3 & 1,2 & 29,4 & 1,51 & 0,71 & 0,64 \\
$\mathrm{Fe}\left(\mathrm{mg} \mathrm{kg}^{-1}\right)$ & 109,5 & 99,7 & 46,9 & 287,1 & 45,0 & 41,1 & 2031,20 & 2,47 & 1,34 \\
$\mathrm{Mn}\left(\mathrm{mg} \mathrm{kg}^{-1}\right)$ & 119,3 & 109,6 & 55,0 & 228,4 & 35,0 & 29,4 & 1218,17 & 0,50 & 0,86 \\
$\mathrm{Zn}\left(\mathrm{mg} \mathrm{kg}^{-1}\right)$ & 22,7 & 22,1 & 15,0 & 43,7 & 4,1 & 17,9 & 16,69 & 7,02 & 1,90 \\
\hline
\end{tabular}

Comparando-se os teores foliares médios dos nutrientes (Tabela 2) com o critério de interpretação pelo método do nível crítico para a cultura do coqueiro híbrido (Tabela 1), os macronutrientes $\mathrm{P}, \mathrm{K}$ e $\mathrm{Ca}$ e os micronutrientes $\mathrm{Fe}, \mathrm{Mn}$ e $\mathrm{Zn}$ situaram-se acima do nível crítico para a cultura do coqueiro híbrido para ambas as referências, parecendo não haver deficiências nutricionais. Para o N, o teor médio de 20,14 $\mathrm{g} \mathrm{kg}^{-1}$ esteve abaixo do nível crítico estabelecido. O teor foliar médio de $\mathrm{Mg}$ de $1,45 \mathrm{~g} \mathrm{~kg}^{-}$ ${ }^{1}$ esteve abaixo dos níveis críticos, constatado em todas as amostras do banco de dados utilizado. Isso pode, possivelmente, ser consequência da baixa eficiência da fertilização magnesiana ou ainda estar associado a efeitos de inibição competitiva com outros cátions no solo. $\mathrm{O}$ teor médio de $\mathrm{S}$ também esteve abaixo dos padrões de referência utilizados para a interpretação da diagnose foliar no coqueiro híbrido, indicando possível deficiência do nutriente no solo.

Para os micronutrientes $\mathrm{B}$ e $\mathrm{Cu}$ os teores foliares médios estiveram acima do nível crítico estabelecido por Silva e Faria (2001), no entanto ambos os nutrientes estiveram abaixo do nível crítico estabelecido por Lins e Viégas (2008) e adotados como padrão de referência para interpretação dos resultados na região de realização do presente estudo.

Para as médias de produtividade de frutos verificou-se que o coeficiente de variação $(\mathrm{CV})$ foi menor no grupo de alta produtividade, comparativamente ao grupo de baixa produtividade, com valores de 3,1 e $27,7 \%$, respectivamente, o que indica menor amplitude na produtividade no grupo de alta produtividade (Tabela 3 ). Os valores dos CVs obtidos para os teores foliares dos nutrientes no grupo de alta ficaram abaixo de $20 \%$ para os macronutrientes e abaixo de $30 \%$ para os micronutrientes. Quanto menor o CV 
menor será a amplitude dos valores dos teores foliares dos nutrientes. Logo, maiores serão os valores dos índices DRIS derivados das funções envolvendo esses nutrientes, resultando em maior possibilidade de indicar desequilíbrios nutricionais para os nutrientes envolvidos (ROCHA et al., 2007; SANTANA et al., 2008).

Tabela 3. Valores médios, mínimos, máximos e coeficiente de variação $(\mathrm{CV})$ para produtividade (coco planta $^{-1}$ ano $\left.^{-1}\right) \mathrm{e}^{-}$ teores foliares de nutrientes em coqueiro híbrido no período de 2001 a 2011 nas subpopulações de alta $(n=15)$ e baixa $(n=119)$ produtividades.

\begin{tabular}{|c|c|c|c|c|c|c|c|c|}
\hline \multirow[t]{2}{*}{ Variável } & \multicolumn{4}{|c|}{ Alta produtividade (PAP) } & \multicolumn{4}{|c|}{ Baixa produtividade (PBP) } \\
\hline & Média & Mínimo & Máximo & $\mathrm{CV}(\%)$ & Média & Mínimo & Máximo & $\mathrm{CV}(\%)$ \\
\hline coco planta & & & & & & & & \\
\hline${ }^{1}$ ano $^{-1}$ & 137 & 131 & 147 & 3,1 & 106 & 10 & 118 & 27,7 \\
\hline $\mathrm{N}\left(\mathrm{g} \mathrm{kg}^{-1}\right)$ & 21,1 & 16,8 & 25,2 & 13,5 & 20,1 & 3,4 & 30,1 & 15,5 \\
\hline $\mathrm{P}\left(\mathrm{g} \mathrm{kg}^{-1}\right)$ & 1,5 & 1,2 & 1,8 & 14,7 & 1,3 & 0,9 & 2,0 & 12,6 \\
\hline $\mathrm{K}\left(\mathrm{g} \mathrm{kg}^{-1}\right)$ & 15,5 & 10,2 & 20,1 & 17,5 & 13,9 & 9,4 & 24,9 & 16,4 \\
\hline $\mathrm{Ca}\left(\mathrm{g} \mathrm{kg}^{-1}\right)$ & 4,2 & 2,8 & 4,9 & 14,1 & 3,5 & 2,1 & 6,5 & 25,8 \\
\hline $\operatorname{Mg}\left(\mathrm{g} \mathrm{kg}^{-1}\right)$ & 1,4 & 1,1 & 1,9 & 16,5 & 1,4 & 0,9 & 2,1 & 17,3 \\
\hline $\mathrm{S}\left(\mathrm{g} \mathrm{kg}^{-1}\right)$ & 1,2 & 1,0 & 1,7 & 19,8 & 1,26 & 0,6 & 2,5 & 25,3 \\
\hline B $\left(\mathrm{mg} \mathrm{kg}^{-1}\right)$ & 15,8 & 9,2 & 22,5 & 25,2 & 15,6 & 5,0 & 36,4 & 33,6 \\
\hline $\mathrm{Cu}\left(\mathrm{mg} \mathrm{kg}^{-1}\right)$ & 4,1 & 2,9 & 5,6 & 24,7 & 4,2 & 2,0 & 8,3 & 29,9 \\
\hline $\mathrm{Fe}\left(\mathrm{mg} \mathrm{kg}^{-1}\right)$ & 124,7 & 62,5 & 189,8 & 29,7 & 108,2 & 46,9 & 287,1 & 42,3 \\
\hline $\operatorname{Mn}\left(\mathrm{mg} \mathrm{kg}^{-1}\right)$ & 109,5 & 70,3 & 157,2 & 18,4 & 120,6 & 55,0 & 228,4 & 30,1 \\
\hline $\mathrm{Zn}\left(\mathrm{mg} \mathrm{kg}^{-1}\right)$ & 22,9 & 19,9 & 27,1 & 9,6 & 22,6 & 15,0 & 43,7 & 18,8 \\
\hline
\end{tabular}

As Tabelas 4 e 5 apresentam as relações selecionadas na PAP baseada nos critérios da maior razão de variância entre o grupo de baixa e o de alta produtividades (Valor "F") e as relações selecionadas com base no critério de cálculo do coeficiente de correlação entre a produtividade e a relação entre os pares de nutrientes (Valor "R"), respectivamente.

Os critérios para a escolha da ordem da razão dos nutrientes (Valor F e Valor R) selecionaram razões diferentes, constatando-se que $56,36 \%$ das relações duais selecionadas (31 relações) foram concordantes (coincidentes) em ambos os métodos de seleção (Tabelas 4 e 5). Serra et al. (2012) desenvolveram normas para a cultura do algodoeiro a partir de banco de dados de 108 lavouras comerciais na região Sul do Mato Grosso. Avaliaram os mesmos critérios de seleção das relações utilizados neste trabalho e constataram que $56 \%$ das relações foram concordantes entre si. Portanto, valores muito próximos aos encontrados neste estudo.

Os coeficientes de variação $(\mathrm{CV})$ das relações selecionadas para compor as normas DRIS pelos critérios empregados foram bastante variáveis. Pelo critério do Valor $\mathrm{F}$ os valores variaram de $6,27 \%(\mathrm{P} /$ N) a $43,63 \%(\mathrm{Cu} / \mathrm{Fe})$, enquanto que pelo critério do Valor $\mathrm{R}$ os valores dos CVs variaram de $6,32 \%(\mathrm{~N} /$ P) a $38,41 \%(\mathrm{~S} / \mathrm{Cu})$. O valor do $\mathrm{CV}$ pode afetar os nutrientes que são considerados mais limitantes com maior frequência, como constatado por Guindani et al. (2009), para a cultura do arroz irrigado no Rio Grande do Sul.

Os valores dos coeficientes de variação das relações entre os nutrientes das normas geradas neste estudo podem ser considerados adequados, uma vez que a literatura sobre o assunto mostra resultados semelhantes. Guindani et al. (2009) desenvolveram normas DRIS para a cultura do arroz irrigado no Rio Grande do Sul e encontraram valores de CVs que variaram de $9,73 \%(\mathrm{~N} / \mathrm{P})$ a $109,86 \%(\mathrm{~B} / \mathrm{Mn})$. Silveira et al. (2005), em trabalho com braquiária, desenvolveram normas DRIS para essa cultura em São Paulo com o uso dos critérios do Valor $\mathrm{F}$ ou $\mathrm{R}$ e encontraram CVs variando de $36,5 \%(\mathrm{~N} / \mathrm{K})$ a $85,7 \%$ $(\mathrm{Mg} / \mathrm{Cu})$. 
Tabela 4. Normas DRIS, média, desvio padrão (DP), coeficiente de variação (CV\%), variância $\left(\mathrm{s}^{2}\right)$ e razão entre as variâncias $-\mathrm{sb}^{2} / \mathrm{sa}^{2}(\mathrm{~F})$ para as relações entre nutrientes na cultura do coqueiro híbrido na população de alta produtividade.

\begin{tabular}{|c|c|c|c|c|c|c|c|c|c|c|c|}
\hline Relação & Média & DP & $\mathrm{CV}$ & $s^{2}$ & $\mathbf{F}$ & Relação & Média & DP & $\mathrm{CV}$ & $s^{2}$ & $\mathbf{F}$ \\
\hline $\mathbf{P} / \mathbf{N}$ & 0,072 & 0,005 & 6,27 & 0,000 & 48,2 & $\mathrm{Mg} / \mathrm{Ca}$ & 0,370 & 0,072 & 19,46 & 0,005 & 2,96 \\
\hline$K / N$ & 0,750 & 0,135 & 17,96 & 0,018 & 8,19 & $\mathrm{Ca} / \mathrm{S}$ & 2,910 & 0,843 & 28,95 & 0,710 & 0,47 \\
\hline $\mathrm{Ca} / \mathbf{N}$ & 0,190 & 0,034 & 17,78 & 0,001 & 9,75 & $\mathrm{~B} / \mathrm{Ca}$ & 3,960 & 1,281 & 32,34 & 1,641 & 3,23 \\
\hline $\mathbf{M g} / \mathbf{N}$ & 0,070 & 0,009 & 12,14 & 0,000 & 17,09 & $\mathrm{Cu} / \mathrm{Ca}$ & 1,035 & 0,102 & 37,04 & 0,147 & 1,68 \\
\hline $\mathbf{S} / \mathbf{N}$ & 0,061 & 0,012 & 19,46 & 0,000 & 13,33 & $\mathrm{Fe} / \mathrm{Ca}$ & 29,410 & 7,667 & 26 & 58,787 & 3,43 \\
\hline $\mathbf{B} / \mathbf{N}$ & 0,760 & 0,226 & 29,75 & 0,051 & 3,19 & $\mathrm{Mn} / \mathrm{Ca}$ & 27,210 & 6,399 & 23,51 & 40,944 & 3,47 \\
\hline $\mathrm{Cu} / \mathbf{N}$ & 0,200 & 0,067 & 33,7 & 0,005 & 2,34 & $\mathrm{Zn} / \mathrm{Ca}$ & 5,700 & 0,809 & 14,19 & 0,654 & 4,48 \\
\hline $\mathrm{Fe} / \mathbf{N}$ & 5,720 & 1,644 & 28,73 & 2,702 & 2,79 & $\mathrm{Mg} / \mathrm{S}$ & 1,180 & 0,223 & 18,89 & 0,050 & 2,76 \\
\hline $\mathbf{M n} / \mathbf{N}$ & 5,230 & 1,113 & 21,28 & 1,239 & 13,32 & $\mathbf{M g} / \mathbf{B}$ & 0,100 & 0,036 & 35,8 & 0,001 & 1,67 \\
\hline $\mathrm{Zn} / \mathbf{N}$ & 1,100 & 0,169 & 15,4 & 0,029 & 10,12 & $\mathrm{Mg} / \mathrm{Cu}$ & 0,390 & 0,146 & 37,41 & 0,001 & 22,31 \\
\hline $\mathbf{P} / \mathbf{K}$ & 0,099 & 0,021 & 21,47 & 0,000 & 12,99 & $\mathrm{Fe} / \mathrm{Mg}$ & 81,450 & 23,000 & 28,23 & 520,079 & 2,21 \\
\hline $\mathbf{P} / \mathbf{C a}$ & 0,376 & 0,064 & 17 & 0,004 & 1,68 & $\mathbf{M n} / \mathbf{M g}$ & 74,560 & 15,673 & 21 & 245,657 & 4,25 \\
\hline $\mathbf{M g} / \mathbf{P}$ & 0,980 & 0,112 & 11,44 & 0,013 & 3,58 & $\mathbf{M g} / \mathbf{Z n}$ & 0,065 & 0,011 & 16,92 & 0,000 & 2,19 \\
\hline $\mathbf{S} / \mathbf{P}$ & 0,850 & 0,131 & 15,35 & 0,017 & 3,98 & $\mathbf{B} / \mathbf{S}$ & 12,560 & 3,486 & 27,75 & 12,152 & 3,49 \\
\hline $\mathbf{B} / \mathbf{P}$ & 10,630 & 3,108 & 29,23 & 9,659 & 2,02 & $\mathrm{~S} / \mathrm{Cu}$ & 0,340 & 0,131 & 38,41 & 0,017 & 1,44 \\
\hline $\mathrm{Cu} / \mathrm{P}$ & 2,790 & 0,944 & 33,83 & 0,891 & 1,15 & $\mathrm{Fe} / \mathrm{S}$ & 93,510 & 20,525 & 21,95 & 421,264 & 5,22 \\
\hline $\mathrm{Fe} / \mathrm{P}$ & 78,950 & 19,368 & 24,54 & 375,137 & 2,52 & $\mathbf{M n} / \mathbf{S}$ & 87,670 & 22,010 & 25,1 & 484,455 & 3,86 \\
\hline $\mathbf{M n} / \mathbf{P}$ & 73,110 & 15,568 & 21,29 & 242,364 & 2,71 & $\mathrm{Zn} / \mathrm{S}$ & 18,270 & 2,383 & 13,04 & 5,678 & 8,00 \\
\hline $\mathbf{Z n} / \mathbf{P}$ & 15,370 & 2,194 & 14,27 & 4,813 & 1,68 & $\mathrm{~B} / \mathrm{Cu}$ & 4,024 & 1,137 & 28,27 & 1,294 & 3,21 \\
\hline $\mathrm{K} / \mathrm{Ca}$ & 3,900 & 0,859 & 22 & 0,738 & 1,80 & $\mathrm{Fe} / \mathrm{B}$ & 8,065 & 3,068 & 38,05 & 9,415 & 2,16 \\
\hline $\mathbf{M g} / \mathrm{K}$ & 0,070 & 0,005 & 6,43 & 0,000 & 22,06 & $\mathbf{M n} / \mathbf{B}$ & 7,360 & 2,350 & 31,92 & 5,521 & 2,79 \\
\hline $\mathbf{S} / \mathbf{K}$ & 0,084 & 0,024 & 28,21 & 0,001 & 0,89 & $\mathrm{Zn} / \mathbf{B}$ & 1,530 & 0,376 & 24,6 & 0,142 & 2,87 \\
\hline $\mathbf{B} / \mathbf{K}$ & 1,059 & 0,417 & 39,34 & 0,174 & 1,42 & $\mathrm{Cu} / \mathrm{Fe}$ & 0,038 & 0,017 & 43,63 & 0,000 & 1,82 \\
\hline $\mathrm{K} / \mathrm{Cu}$ & 4,100 & 1,265 & 30,85 & 1,600 & 1,13 & $\mathrm{Mn} / \mathrm{Cu}$ & 28,410 & 9,104 & 32 & 82,880 & 2,65 \\
\hline $\mathrm{Fe} / \mathrm{K}$ & 7,870 & 2,561 & 32,53 & 6,556 & 2,12 & $\mathrm{Zn} / \mathrm{Cu}$ & 5,990 & 1,690 & 28,2 & 2,854 & 1,71 \\
\hline $\mathbf{M n} / \mathbf{K}$ & 7,150 & 1,833 & 25,63 & 3,359 & 2,82 & $\mathbf{M n} / \mathbf{F e}$ & 0,998 & 0,377 & 37,74 & 0,142 & 2,78 \\
\hline \multirow[t]{2}{*}{$\mathrm{Zn} / \mathbf{K}$} & 1,510 & 0,327 & 21,65 & 0,107 & 1,42 & $\mathrm{Zn} / \mathrm{Fe}$ & 0,210 & 0,071 & 33,57 & 0,005 & 2,21 \\
\hline & & & & & & $\mathbf{M n} / \mathbf{Z n}$ & 4,790 & 0,990 & 20,67 & 0,980 & 1,96 \\
\hline
\end{tabular}

Tabela 5. Normas DRIS, média, desvio padrão (DP), coeficiente de variação (CV\%), variância ( $\left.\mathrm{s}^{2}\right)$ e coeficiente de correlação $(\mathrm{R})$ considerando o fator $\mathrm{R}$ para as relações entre nutrientes na cultura do coqueiro híbrido na população de alta produtividade.

\begin{tabular}{cccccccccccc}
\hline Relação & Média & $\mathbf{D P}$ & $\mathbf{C V}$ & $\mathbf{s}^{\mathbf{2}}$ & $\mathbf{R}$ & $\mathbf{R e l a c ̧ a ̃ o}$ & Média & $\mathbf{D P}$ & $\mathbf{C V}$ & $\mathbf{s}^{\mathbf{2}}$ & $\mathbf{R}$ \\
\hline $\mathbf{N} / \mathbf{P}$ & 13,980 & 0,8836 & 6,32 & 0,780 & 0,47 & $\mathbf{C a} / \mathbf{M g}$ & 2,790 & 0,5118 & 18,34 & 0,261 & 0,13 \\
$\mathbf{K} / \mathbf{N}$ & 0,750 & 0,1347 & 17,96 & 0,018 & 0,47 & $\mathbf{S} / \mathbf{C a}$ & 0,376 & 0,1286 & 34,20 & 0,016 & $-0,06$ \\
$\mathbf{C a} / \mathbf{N}$ & 0,190 & 0,0338 & 17,78 & 0,001 & 0,22 & $\mathbf{B} / \mathbf{C a}$ & 3,960 & 1,2808 & 32,34 & 1,640 & $-0,31$ \\
$\mathbf{N} / \mathbf{M g}$ & 14,350 & 1,8023 & 12,56 & 3,248 & $-0,11$ & $\mathbf{C a} / \mathbf{C u}$ & 1,070 & 0,3447 & 32,21 & 0,118 & $-0,17$ \\
$\mathbf{N} / \mathbf{S}$ & 16,910 & 3,3662 & 19,90 & 11,331 & $-0,23$ & $\mathbf{F e} / \mathbf{C a}$ & 29,410 & 7,6672 & 26,00 & 58,78 & $-0,57$ \\
$\mathbf{N} / \mathbf{B}$ & 1,430 & 0,4448 & 31,00 & 0,197 & 0,21 & $\mathbf{M n} / \mathbf{C a}$ & 27,210 & 6,3987 & 23,51 & 40,94 & 0,33 \\
$\mathbf{N} / \mathbf{C u}$ & 5,610 & 1,9392 & 34,56 & 3,760 & $-0,21$ & $\mathbf{C a} / \mathbf{Z n}$ & 0,180 & 0,0238 & 13,22 & 0,000 & 0,13 \\
$\mathbf{F e} / \mathbf{N}$ & 5,720 & 1,6436 & 28,73 & 2,701 & $-0,38$ & $\mathbf{S} / \mathbf{M g}$ & 0,870 & 0,1554 & 17,86 & 0,024 & $-0,31$ \\
$\mathbf{M n} / \mathbf{N}$ & 5,230 & 1,1132 & 21,28 & 1,239 & 0,50 & $\mathbf{M g} / \mathbf{B}$ & 0,100 & 0,0358 & 35,80 & 0,001 & 0,20 \\
$\mathbf{Z n} / \mathbf{N}$ & 1,100 & 0,1695 & 15,40 & 0,028 & 0,15 & $\mathbf{M g} / \mathbf{C u}$ & 0,390 & 0,1459 & 37,41 & 0,001 & $-0,20$ \\
$\mathbf{K} / \mathbf{P}$ & 10,500 & 2,1515 & 20,49 & 4,629 & 0,56 & $\mathbf{F e} / \mathbf{M g}$ & 81,450 & 23,0000 & 28,23 & 520,0 & $-0,42$ \\
$\mathbf{P} / \mathbf{C a}$ & 0,376 & 0,0641 & 17,00 & 0,004 & $-0,39$ & $\mathbf{M g} / \mathbf{M n}$ & 0,014 & 0,0031 & 22,07 & 0,000 & $-0,44$
\end{tabular}


Tabela 5. Continuação.

\begin{tabular}{cccccccccccc}
\hline Relação & Média & $\mathbf{D P}$ & $\mathbf{C V}$ & $\mathbf{S}^{\mathbf{2}}$ & $\mathbf{R}$ & $\mathbf{R e l a c ̧ a ̃ o}$ & Média & $\mathbf{D P}$ & $\mathbf{C V}$ & $\begin{array}{c}\mathbf{s}^{\mathbf{2}} \\
\mathbf{R}\end{array}$ \\
$\mathbf{P} / \mathbf{M g}$ & 1,027 & 0,1209 & 11,77 & 0,014 & $-0,37$ & $\mathbf{M g} / \mathbf{Z n}$ & 0,065 & 0,0110 & 16,92 & 0,000 & $-0,04$ \\
$\mathbf{P} / \mathbf{S}$ & 1,200 & 0,1922 & 16,01 & 0,036 & 0,10 & $\mathbf{B} / \mathbf{S}$ & 12,560 & 3,4858 & 27,75 & 12,15 & $-0,08$ \\
$\mathbf{P} / \mathbf{B}$ & 0,102 & 0,0314 & 30,69 & 0,001 & 0,10 & $\mathbf{S} / \mathbf{C u}$ & 0,340 & 0,1306 & 38,41 & 0,017 & $-0,38$ \\
$\mathbf{P} / \mathbf{C u}$ & 0,403 & 0,1476 & 36,58 & 0,021 & $-0,31$ & $\mathbf{F e} / \mathbf{S}$ & 93,510 & 20,5247 & 21,95 & 421,2 & $-0,30$ \\
$\mathbf{F e / P}$ & 78,950 & 19,3684 & 24,54 & 375,13 & $-0,32$ & $\mathbf{M n} / \mathbf{S}$ & 87,670 & 22,0100 & 25,10 & 484,4 & 0,63 \\
$\mathbf{M n} / \mathbf{P}$ & 73,110 & 15,5680 & 21,29 & 242,36 & 0,64 & $\mathbf{S} / \mathbf{Z n}$ & 0,055 & 0,0076 & 13,80 & 0,000 & $-0,48$ \\
$\mathbf{Z n / P}$ & 15,370 & 2,1937 & 14,27 & 4,812 & 0,34 & $\mathbf{B} / \mathbf{C u}$ & 4,024 & 1,1374 & 28,27 & 1,293 & $-0,47$ \\
$\mathbf{K} / \mathbf{C a}$ & 3,900 & 0,8588 & 22,00 & 0,737 & 0,27 & $\mathbf{F e} / \mathbf{B}$ & 8,065 & 3,0683 & 38,05 & 9,414 & $-0,17$ \\
$\mathbf{M g} / \mathbf{K}$ & 0,070 & 0,0045 & 6,43 & 0,000 & $-0,47$ & $\mathbf{M n} / \mathbf{B}$ & 7,360 & 2,3496 & 31,92 & 5,520 & 0,60 \\
$\mathbf{K} / \mathbf{S}$ & 12,640 & 3,1761 & 25,12 & 10,088 & 0,57 & $\mathbf{B} / \mathbf{Z n}$ & 0,688 & 0,1623 & 23,60 & 0,026 & $-0,32$ \\
$\mathbf{K} / \mathbf{B}$ & 1,070 & 0,3829 & 35,78 & 0,146 & 0,45 & $\mathbf{F e} / \mathbf{C u}$ & 32,390 & 16,4957 & 50,92 & 272,1 & $-0,46$ \\
$\mathbf{K} / \mathbf{C u}$ & 4,100 & 1,2648 & 30,85 & 1,599 & 0,05 & $\mathbf{M n} / \mathbf{C u}$ & 28,410 & 9,1040 & 32,00 & 82,88 & 0,18 \\
$\mathbf{F e} / \mathbf{K}$ & 7,870 & 2,5605 & 32,53 & 6,556 & $-0,57$ & $\mathbf{Z n / C u}$ & 5,990 & 1,6895 & 28,20 & 2,854 & $-0,24$ \\
$\mathbf{K} / \mathbf{M n}$ & 0,150 & 0,0367 & 24,46 & 0,001 & $-0,08$ & $\mathbf{M n} / \mathbf{F e}$ & 0,998 & 0,3767 & 37,74 & 0,141 & 0,56 \\
$\mathbf{K} / \mathbf{Z n}$ & 0,690 & 0,1265 & 18,34 & 0,016 & 0,43 & $\mathbf{F e} / \mathbf{Z n}$ & 5,230 & 1,4560 & 27,84 & 2,120 & $-0,48$ \\
& & & & & & $\mathbf{M n} / \mathbf{Z n}$ & 4,790 & 0,9901 & 20,67 & 0,980 & 0,50 \\
\hline
\end{tabular}

Para frutíferas perenes se destaca o trabalho de Santana et al. (2008), cujo objetivo foi desenvolver normas DRIS para a cultura da laranja no Estado de Goiás, empregando os critérios do Valor F, os quais encontraram valores de $\mathrm{CV}$ que variaram de $19,3 \%(\mathrm{~N} / \mathrm{P})$ a $159,3(\mathrm{Cu} / \mathrm{Mn})$. Santos et al. (2004), ao desenvolver normas DRIS para a cultura do coqueiro anão verde no Estado do Rio de Janeiro, encontraram valores de CVs que variaram de $5,8 \%(\mathrm{~N} /$ P) a $69,4 \%(\mathrm{Fe} / \mathrm{B})$ nas relações selecionadas que compuseram as normas.

Nas 55 relações selecionadas por meio do critério do Valor F (Tabela 4) verificou-se que 14 relações $(\mathrm{Cu} / \mathrm{P} ; \mathrm{B} / \mathrm{K} ; \mathrm{K} / \mathrm{Cu} ; \mathrm{Fe} / \mathrm{K} ; \mathrm{B} / \mathrm{Ca} ; \mathrm{Cu} / \mathrm{Ca} ; \mathrm{Mg} /$ $\mathrm{B} ; \mathrm{Mg} / \mathrm{Cu} ; \mathrm{S} / \mathrm{Cu} ; \mathrm{Fe} / \mathrm{B} ; \mathrm{Mn} / \mathrm{B} ; \mathrm{Cu} / \mathrm{Fe} ; \mathrm{Mn} / \mathrm{Fe}$ e $\mathrm{Zn} /$ Fe) apresentaram CV acima de 30\%. Constatou-se, ainda, que em todas as relações em que isso aconteceu houve envolvimento de micronutrientes, com destaque para os elementos $\mathrm{B}, \mathrm{Cu}$ e Fe em decorrência dos maiores valores de $\mathrm{CV}$ dos teores foliares no grupo de alta produtividade (Tabela 3 ) serem dos nutrientes $\mathrm{B}, \mathrm{Cu}$ e $\mathrm{Fe}$, com valores de $\mathrm{CV}$ de 25,2, 24,7 e $29,7 \%$, respectivamente.

Nas 55 relações selecionadas por meio do critério do Valor $\mathrm{R}$ (Tabela 5) verificou-se que 17 relações (N/Cu; P/B; P/Cu; K/B; K/Cu; Fe/K; S/Ca; $\mathrm{B} / \mathrm{Ca} ; \mathrm{Ca} / \mathrm{Cu} ; \mathrm{Mg} / \mathrm{B} ; \mathrm{Mg} / \mathrm{Cu} ; \mathrm{S} / \mathrm{Cu} ; \mathrm{Fe} / \mathrm{B} ; \mathrm{Mn} / \mathrm{B} ; \mathrm{Fe} /$ $\mathrm{Cu} ; \mathrm{Mn} / \mathrm{Cu} ; \mathrm{Mn} / \mathrm{Fe}$ ) apresentaram $\mathrm{CV}$ acima de $30 \%$. Em 94,12\% dessas relações (16 relações) houve envolvimento de micronutrientes, da mesma forma que o observado para o critério Valor $\mathrm{F}$, em que os micronutrientes $\mathrm{B}, \mathrm{Cu}$ e $\mathrm{Fe}$ também estiveram envolvidos em quase todas as relações onde se observou CV acima de $30 \%$

Pelo critério de seleção das relações duais com uso do Valor R, a correlação entre pares de nu- trientes e a produtividade apresentou valores em módulo mínimos de $\mathrm{R}=0,05(\mathrm{~K} / \mathrm{Cu})$ e máximo de $\mathrm{R}=$ 0,64 (Mn/P). Nachtigall e Dechen (2007), em estudo com 70 pomares de macieira no Rio Grande do Sul, desenvolveram normas DRIS para a cultura e encontraram valores em módulo mínimos de $\mathrm{R}=0,03(\mathrm{Mn} /$ $\mathrm{N})$ e máximo de $\mathrm{R}=0,44(\mathrm{~B} / \mathrm{Fe})$. Mourão Filho et al (2002) encontraram valores mínimos de $\mathrm{R}=0,05$ $(\mathrm{Mn} / \mathrm{Cu})$ e máximo de $\mathrm{R}=0,83(\mathrm{Mg} / \mathrm{Ca})$ ao estabelecerem normas DRIS em pomares comerciais de laranjeira Valência com dados coletados no período de 1994 a 1998 ao utilizarem o critério do Valor R para a seleção das relações diretas ou inversas. Os valores encontrados no presente estudo apresentam amplitudes semelhantes às observadas na literatura para outras culturas, conforme citações anteriores.

É importante que as normas DRIS selecionadas estejam baseadas em uma distribuição normal, caso contrário a média calculada para uma relação entre nutrientes (normas DRIS) pode diferir do verdadeiro valor obtido em uma lavoura de produtividade desejada (WALWORTH; SUMNER, 1987). Essa mesma preocupação com a normalidade dos dados das normas foi considerada por Ramakrishna et al. (2009), ao selecionarem relações que apresentaram CV menor ou igual a $35 \%$.

Observou-se que a normalidade das relações selecionadas em função do fator $\mathrm{F}$ avaliadas pelo teste de Shapiro e Wilk (1965) (Tabela 6) indicou que somente $9,0 \%$ (cinco relações de 55 selecionadas - $\mathrm{Cu} / \mathrm{N} ; \mathrm{Cu} / \mathrm{P} ; \mathrm{Zn} / \mathrm{K} ; \mathrm{Cu} / \mathrm{Ca}$ e $\mathrm{Zn} / \mathrm{Fe}$ ) não apresentaram distribuição normal. Para as relações selecionadas em função do fator $\mathrm{R}$ (Tabela 7) apenas 5,4\% (três relações de 55 selecionadas $-\mathrm{N} / \mathrm{Cu} ; \mathrm{P} / \mathrm{Cu}$ e $\mathrm{Fe} /$ $\mathrm{Cu}$ ) não apresentaram distribuição normal. Isso pode ser um indicador de qualidade das normas geradas 
por ambos os critérios de seleção das razões entre os nutrientes.

Destaca-se, ainda, que para as oito relações selecionadas por ambos os critérios de seleção (Fator F e Fator R) que não apresentaram distribuição normal o nutriente $\mathrm{Cu}$ esteve presente em seis relações $(75 \%)$. Isso indica que esse elemento $(\mathrm{Cu})$ apresenta tendência em comprometer a normalidade das relações duais entre os nutrientes. Outrossim, verificou- se que em todas as relações entre nutrientes que não apresentaram normalidade os CVs estiveram sempre acima de $30 \%$, com exceção da relação $\mathrm{Zn} / \mathrm{K}$ (CV 21,65\%) (Tabelas 4 e 5). Destaca-se a relação $\mathrm{Fe} / \mathrm{Cu}$ que apresentou o maior CV $(50,92 \%)$, decorrente, possivelmente, dos teores individuais de Fe e $\mathrm{Cu}$ apresentar alto valor de $\mathrm{CV}$ em seus teores individuais (Tabela 2).

Tabela 6. Teste de normalidade Shapiro - Wilk para as relações selecionadas em função do Fator F.

\begin{tabular}{|c|c|c|c|c|c|c|c|c|}
\hline Relação & Teste W & P-valor & Relação & Teste W & P-valor & Relação & Teste W & P-valor \\
\hline $\mathbf{P} / \mathbf{N}$ & $0,97^{*}$ & 0,88 & $\mathrm{~K} / \mathrm{Ca}$ & $0,95 *$ & 0,58 & $\mathrm{Mn} / \mathrm{Mg}$ & $0,91 *$ & 0,18 \\
\hline$K / N$ & $0,95 *$ & 0,65 & $\mathrm{Mg} / \mathrm{K}$ & $0,97 *$ & 0,88 & $\mathbf{M g} / \mathbf{Z n}$ & $0,95^{*}$ & 0,53 \\
\hline $\mathrm{Ca} / \mathrm{N}$ & $0,94 *$ & 0,50 & $\mathbf{S} / \mathbf{K}$ & $0,90^{*}$ & 0,12 & $\mathrm{~B} / \mathrm{S}$ & $0,90^{*}$ & 0,12 \\
\hline $\mathbf{M g} / \mathbf{N}$ & $0,94 *$ & 0,47 & $\mathbf{B} / \mathbf{K}$ & $0,90 *$ & 0,11 & $\mathrm{~S} / \mathrm{Cu}$ & $0,91 *$ & 0,14 \\
\hline $\mathbf{S} / \mathbf{N}$ & $0,97^{*}$ & 0,90 & $\mathrm{~K} / \mathrm{Cu}$ & $0,98^{*}$ & 0,99 & $\mathrm{Fe} / \mathrm{S}$ & $0,90 *$ & 0,11 \\
\hline $\mathbf{B} / \mathbf{N}$ & $0,92 *$ & 0,22 & $\mathrm{Fe} / \mathrm{K}$ & $0,96^{*}$ & 0,84 & $\mathbf{M n} / \mathbf{S}$ & $0,95^{*}$ & 0,61 \\
\hline $\mathrm{Cu} / \mathbf{N}$ & $0,87^{\mathrm{ns}}$ & 0,03 & $\mathbf{M n} / \mathbf{K}$ & $0,91 *$ & 0,18 & $\mathrm{Zn} / \mathrm{S}$ & $0,92 *$ & 0,2 \\
\hline $\mathrm{Fe} / \mathrm{N}$ & $0,92 *$ & 0,41 & $\mathbf{Z n} / \mathbf{K}$ & $0,87^{\mathrm{ns}}$ & 0,03 & $\mathrm{~B} / \mathrm{Cu}$ & $0,92 *$ & 0,23 \\
\hline $\mathbf{M n} / \mathbf{N}$ & $0,96^{*}$ & 0,74 & $\mathrm{Mg} / \mathrm{Ca}$ & $0,91 *$ & 0,14 & $\mathrm{Fe} / \mathrm{B}$ & $0,94 *$ & 0,43 \\
\hline $\mathrm{Zn} / \mathbf{N}$ & $0,94 *$ & 0,48 & $\mathrm{Ca} / \mathrm{S}$ & $0,91 *$ & 0,18 & $\mathbf{M n} / \mathbf{B}$ & $0,97 *$ & 0,86 \\
\hline $\mathbf{P} / \mathbf{K}$ & $0,96^{*}$ & 0,10 & $\mathbf{B} / \mathbf{C a}$ & $0,88^{*}$ & 0,06 & $\mathrm{Zn} / \mathrm{B}$ & $0,90^{*}$ & 0,11 \\
\hline $\mathbf{P} / \mathbf{C a}$ & $0,92 *$ & 0,25 & $\mathrm{Cu} / \mathrm{Ca}$ & $0,87^{\mathrm{ns}}$ & 0,04 & $\mathrm{Cu} / \mathrm{Fe}$ & $0,95 *$ & 0,65 \\
\hline $\mathrm{Mg} / \mathrm{P}$ & $0,95^{*}$ & 0,62 & $\mathrm{Fe} / \mathrm{Ca}$ & $0,97 *$ & 0,94 & $\mathbf{M n} / \mathbf{C u}$ & $0,95^{*}$ & 0,6 \\
\hline $\mathbf{S} / \mathbf{P}$ & $0,94 *$ & 0,50 & $\mathbf{M n} / \mathbf{C a}$ & $0,92 *$ & 0,2 & $\mathrm{Zn} / \mathrm{Cu}$ & $0,96^{*}$ & 0,81 \\
\hline $\mathbf{B} / \mathbf{P}$ & $0,96^{*}$ & 0,69 & $\mathrm{Zn} / \mathrm{Ca}$ & $0,94 *$ & 0,42 & $\mathbf{M n} / \mathbf{F e}$ & $0,90^{*}$ & 0,11 \\
\hline $\mathbf{C u} / \mathbf{P}$ & $0,87^{\mathrm{ns}}$ & 0,04 & $\mathbf{M g} / \mathrm{S}$ & $0,94^{*}$ & 0,51 & $\mathrm{Zn} / \mathrm{Fe}$ & $0,84^{\mathrm{ns}}$ & 0,01 \\
\hline $\mathrm{Fe} / \mathrm{P}$ & $0,93 *$ & 0,31 & $\mathrm{Mg} / \mathrm{B}$ & $0,94 *$ & 0,49 & $\mathbf{M n} / \mathbf{Z n}$ & $0,95^{*}$ & 0,62 \\
\hline $\mathbf{M n} / \mathbf{P}$ & $0,92 *$ & 0,25 & $\mathrm{Mg} / \mathrm{Cu}$ & $0,90 *$ & 0,11 & & & \\
\hline $\mathbf{Z n} / \mathbf{P}$ & $0,98 *$ & 0,96 & $\mathrm{Fe} / \mathrm{Mg}$ & $0,98 *$ & 0,99 & & & \\
\hline
\end{tabular}

${ }^{*}$ significativo $(p<0,05) ;{ }^{\text {ns }}$ - não significativo $(p>0,05)$ pelo teste $F$.

Tabela 7. Teste de normalidade Shapiro - Wilk para as relações selecionadas em função do Fator R.

\begin{tabular}{ccccccccc}
\hline Relação & Teste W & P-valor & Relação & Teste W & P-valor & Relação & Teste W & P-valor \\
\hline $\mathbf{N} / \mathbf{P}$ & $0,97^{*}$ & 0,86 & $\mathbf{K} / \mathbf{C a}$ & $0,95^{*}$ & 0,58 & $\mathbf{M g} / \mathbf{M n}$ & $0,89^{*}$ & 0,08 \\
$\mathbf{K} / \mathbf{N}$ & $0,95^{*}$ & 0,65 & $\mathbf{M g} / \mathbf{K}$ & $0,97^{*}$ & 0,88 & $\mathbf{M g} / \mathbf{Z n}$ & $0,95^{*}$ & 0,53 \\
$\mathbf{C a} / \mathbf{N}$ & $0,94^{*}$ & 0,5 & $\mathbf{K} / \mathbf{S}$ & $0,98^{*}$ & 0,58 & $\mathbf{B} / \mathbf{S}$ & $0,90^{*}$ & 0,12
\end{tabular}


Tabela 7. Continuação.

\begin{tabular}{|c|c|c|c|c|c|c|c|c|}
\hline Relação & Teste W & P-valor & Relação & Teste W & P-valor & Relação & Teste W & P-valor \\
\hline N/Mg & $0,93 *$ & 0,28 & $\mathrm{~K} / \mathrm{B}$ & $0,96^{*}$ & 0,58 & $\mathrm{~S} / \mathrm{Cu}$ & $0,91 *$ & 0,14 \\
\hline $\mathbf{N} / \mathbf{S}$ & $0,95^{*}$ & 0,63 & $\mathrm{~K} / \mathrm{Cu}$ & $0,98 *$ & 0,99 & $\mathrm{Fe} / \mathrm{S}$ & $0,90^{*}$ & 0,11 \\
\hline N/B & $0,88^{*}$ & 0,064 & $\mathrm{Fe} / \mathrm{K}$ & $0,96 *$ & 0,84 & $\mathbf{M n} / \mathbf{S}$ & $0,95 *$ & 0,86 \\
\hline $\mathbf{N} / \mathbf{C u}$ & $0,85^{\mathrm{ns}}$ & 0,018 & K/Mn & $0,95 *$ & 0,55 & $\mathbf{S} / \mathbf{Z n}$ & $0,90 *$ & 0,25 \\
\hline $\mathrm{Fe} / \mathrm{N}$ & $0,92 *$ & 0,41 & $\mathrm{~K} / \mathbf{Z n}$ & $0,97 *$ & 0,93 & $\mathrm{~B} / \mathrm{Cu}$ & $0,92 *$ & 0,23 \\
\hline $\mathbf{M n} / \mathbf{N}$ & $0,96 *$ & 0,74 & $\mathrm{Ca} / \mathrm{Mg}$ & $0,94 *$ & 0,47 & $\mathrm{Fe} / \mathrm{B}$ & $0,94 *$ & 0,43 \\
\hline $\mathrm{Zn} / \mathbf{N}$ & $0,84^{*}$ & 0,48 & $\mathrm{~S} / \mathrm{Ca}$ & $0,93 *$ & 0,29 & $\mathbf{M n} / \mathbf{B}$ & $0,97 *$ & 0,86 \\
\hline $\mathbf{K} / \mathbf{P}$ & $0,92 *$ & 0,58 & $\mathbf{B} / \mathbf{C a}$ & $0,88^{*}$ & 0,06 & $\mathbf{B} / \mathbf{Z n}$ & $0,93 *$ & 0,37 \\
\hline $\mathrm{P} / \mathrm{Ca}$ & $0,92 *$ & 0,25 & $\mathrm{Ca} / \mathrm{Cu}$ & $0,96 *$ & 0,82 & $\mathrm{Fe} / \mathrm{Cu}$ & $0,86^{\mathrm{ns}}$ & 0,02 \\
\hline P/Mg & $0,93 *$ & 0,29 & $\mathrm{Fe} / \mathrm{Ca}$ & $0,97 *$ & 0,94 & $\mathrm{Mn} / \mathrm{Cu}$ & $0,95 *$ & 0,60 \\
\hline $\mathbf{P} / \mathbf{S}$ & $0,91 *$ & 0,18 & $\mathrm{Mn} / \mathrm{Ca}$ & $0,92 *$ & 0,20 & $\mathrm{Zn} / \mathrm{Cu}$ & $0,96^{*}$ & 0,81 \\
\hline $\mathbf{P} / \mathbf{B}$ & $0,93 *$ & 0,36 & $\mathrm{Ca} / \mathrm{Zn}$ & $0,97 *$ & 0,93 & $\mathbf{M n} / \mathbf{F e}$ & $0,90 *$ & 0,11 \\
\hline $\mathrm{P} / \mathrm{Cu}$ & $0,84^{\mathrm{ns}}$ & 0,02 & S/Mg & $0,98 *$ & 0,98 & $\mathrm{Fe} / \mathrm{Zn}$ & $0,96 *$ & 0,80 \\
\hline $\mathrm{Fe} / \mathrm{P}$ & $0,93 *$ & 0,31 & $\mathrm{Mg} / \mathrm{B}$ & $0,94 *$ & 0,49 & $\mathbf{M n} / \mathbf{Z n}$ & $0,95 *$ & 0,62 \\
\hline $\mathbf{M n} / \mathbf{P}$ & $0,92 *$ & 0,25 & $\mathrm{Mg} / \mathrm{Cu}$ & $0,90 *$ & 0,11 & & & \\
\hline $\mathbf{Z n} / \mathbf{P}$ & $0,98^{*}$ & 0,96 & $\mathrm{Fe} / \mathrm{Mg}$ & $0,98 *$ & 0,99 & & & \\
\hline
\end{tabular}

${ }^{*}$ significativo $(\mathrm{p}<0,05) ;{ }^{\text {ns }}$ - não significativo $(\mathrm{p}>0,05)$ pelo teste $\mathrm{F}$.

Tem sido relativamente comum a constatação de elevados valores de $\mathrm{CV}$ para as relações entre nutrientes envolvendo micronutrientes que compõem as normas DRIS. Pardo et al. (2013), em trabalho com desenvolvimento de normas DRIS para a seringueira (Hevea brasiliensis), utilizando dados coletados durante um ano, em área de produção comercial com 12 anos na Colômbia, verificaram elevados valores de CVs para algumas relações entre micronutrientes, quais sejam: $\mathrm{Fe} / \mathrm{P}(50,38 \%) ; \mathrm{K} / \mathrm{B}(53,87 \%)$; $\mathrm{Ca} / \mathrm{Fe}(53,10 \%) ; \mathrm{Cu} / \mathrm{Mn}(67,13 \%) ; \mathrm{Mn} / \mathrm{Cu}(60,5 \%)$; e $\mathrm{B} / \mathrm{Fe}(82 \%)$. Os autores ora mencionados atribuem os altos valores de CVs nas relações envolvendo micronutrientes à maior amplitude de variação dos teores individuais desses nutrientes. $\mathrm{O}$ trabalho de Farnezi et al. (2009) estabelecendo normas DRIS em lavouras de café no Vale do Jequitinhonha, em Minas Gerais, também constatou altos valores de CVs nas relações envolvendo os micronutrientes $\mathrm{Fe}, \mathrm{Mn}$ e $\mathrm{Cu}$, indicando que estes nutrientes apresentaram maior variação em torno da média.

No presente estudo, as normas geradas não foram submetidas às transformações em razão do grande percental de normalidade verificado no teste Shapiro - Wilk, observando-se, ainda, embasamento na literatura. De acordo Politi et al. (2013), os quais compararam diagnósticos do estado nutricional de mangueira na região do Vale do São Francisco pelos métodos CND e DRIS, assim como normas com e sem transformação logarítmica, constataram que houve desempenho semelhante na avaliação do estado nutricional da mangueira utilizando normas com e sem transformação logarítmica. Serra et al. (2012) avaliaram o estado nutricional do algodoeiro por meio do DRIS, também testaram dois conjuntos de normas, com e sem transformação das relações entre os nutrientes, e concluíram que os dois critérios para a seleção das normas foram eficientes em diagnosticar o estado nutricional das plantas, além da concordância entre os métodos na avaliação do estado nutricional entre 92,6 e $100 \%$

\section{CONCLUSÕES}


Houve maior variação nos teores de Fe, Mn e B no banco de dados.

Os teores foliares de $\mathrm{Mg}$ se apresentaram abaixo dos níveis críticos foliares para todo o banco de dados.

$\mathrm{O} \mathrm{Cu}$ comprometeu a normalidade das relações duais entre nutrientes.

E os diferentes conjuntos de normas gerados irá permitir a escolha do método que melhor se ajuste a cultura do coqueiro híbrido em futuros estudos com o método DRIS com essa cultura.

\section{AGREDECIMENTOS}

Os autores agradecem a Empresa SOCOCO Agroindústria da Amazônia pelo apoio técnico e operacional para a realização do presente estudo, como também ao Engenheiro Agrônomo Dr. Paulo Manoel Pontes Lins, Diretor de Pesquisa e Desenvolvimento do grupo SOCOCO pelo apoio e disponibilidade dos dados.

\section{REFERÊNCIAS}

BEAUFILS, E. R. Diagnosis and Recommendation Integrated System (DRIS): a general scheme for experimentation and calibration based on principles develop from research in plant nutrition. 1. ed. South Africa: Pietermaritzburg, 1973. 132 p. (Soil Science Bulletin, 1)

CASTAMANN, A. et al. Diagnosis and recommendation integrated system (DRIS) of soybean oil content. Revista Brasileira de Ciência do Solo, Viçosa, v. 36, n. 6, p. 1820-1827. 2012.

DIAS, J. R. M. et al. Estabelecimento de normas DRIS para o cupuaçueiro na região Amazônica. Revista Caatinga, Mossoró, v. 23, n. 4, p. 121-128, 2010 .

DIAS, J. R. M. et al. Normas DRIS multivariadas para avaliação do estado nutricional de laranjeira "Pera" no estado do Amazonas. Ciência Agronômica, Fortaleza, v. 44, n. 2, p. 251-259, 2013.

EMBRAPA. Sistema brasileiro de classificação de solos. 3. ed. Brasília: Embrapa/CNPS, 2013. 353 p.

FARNEZI, M. M. M.; SILVA, E. B.; GUIMARÃES, P. T. G. Diagnose nutricional de cafeeiros da região do Alto do Jequitinhonha (MG): Normas DRIS e faixas críticas de nutrientes. Revista Brasileira de Ciência do Solo, Viçosa, v. 33, n. 4, p. 969-978, 2009.

GUINDANI, R. R. H. P.; ANGHINONI, I.; NACH-
TIGALL, G. R. DRIS na avaliação do estado nutricional do arroz irrigado por inundação Revista Brasileira de Ciência do Solo, Viçosa, v. 33, n. 1, p. 109 $118,2009$.

LETZSCH, W. S. Computer program for selection of norms for use in the diagnosis and recommendation integrated system (DRIS). Communications in Soil Science and Plant Analysis, Madison, v. 16, n. 4, p. 339-347, 1985.

LINS, P. M. P.; VIÉGAS, I. J. M. Adubação do coqueiro no Pará. 1. ed. Belém: Embrapa Amazônia Oriental, 2008. 28 p. (Documentos, 350).

LINS, P. M. P.; NETO, J. T. F.; MULLER, A. A. Avaliação de híbridos de coqueiro (Cocos nucifera L.) para produção de frutos e de albúmen sólido fresco. Revista. Revista Brasileira de Fruticultura, Jaboticabal, v. 25, n. 3, p. 468-470, 2003.

MALAVOLTA, E.; VITTI, G. C.; OLIVEIRA, S. A. Avaliação do estado nutricional das plantas: princípios e aplicações. 2. ed. Piracicaba: POTAFOS, 1997. 319 p.

MANCIOT, R.; OLLAGNIER, M.; OCHS, R. Nutricion mineral y fertilizacion de cocotero en lo mundo. Oléagineux, Paris, v. 35, n. 1, p. 13-27. 1980.

MOHANDAS, S. Effect of NPK fertilizer levels on mineral nutrition and yield of hybrid (Tall $x$ Dwarf) coconut. Madras Agricultural Journal, Tamil Nadu, v. 99, n. 1-3, p. 87-91. 2012.

MOURÃO FILHO, F. A. A.; AZEVEDO, J. C.; NICK, J. A. Funções e ordem da razão dos nutrientes no estabelecimento de normas DRIS em laranja "Valência". Pesquisa Agropecuária Brasileira, Brasília, v. 37, n. 2, p. 185-192, 2002.

NACHTIGALL, G. M.; DECHEN, A. R. Dris norms for evaluating the nutricional state of Apple tree. Scientia Agrícola, Piracicaba, v. 64, n. 3, p. $282-$ 287, 2007

PARDO, C. E.; TAMAYO, J. H. C.; ARGUELLO, O. Establishment of norms for the nutritional diagnosis of rubber (Hevea brasiliensis Muell Arg.) clone RRIM 600 on the Earstern Plains of Colombia. Agronomia Colombiana, Bogotá, v. 31, n. 2, p. 215 $-222,2013$.

POLITI, L. S. et al. Estado nutricional de mangueiras determinado pelos métodos DRIS e CND. Revista Brasileira de Engenharia Agrícola e Ambiental, Campina Grande, v. 17, n. 1, p. 11-18, 2013.

RAMAKRISHNA, A.; BAILEY, J. S.; KIRCHHOF, G. A preliminary diagnosis and recommendation 
integrated system (DRIS) model for diagnosing the nutrient status of sweet potato (Ipomoea batatas). Plant and Soil, New York, v. 316, n. 1-2, p. 107116, 2009.

ROCHA, A. C. et al. Normas DRIS para cultura do milho semeado em espaçamento reduzido na região de Hidrolândia, GO, Brasil. Bioscience Journal, Goiânia, v. 23, n. 4, p. 50-60, 2007.

SANTANA, J. G. et al. da. Normas DRIS para interpretação de análises de folha e solo, em laranjeira pêra, na região central de Goiás. Pesquisa Agropecuária Tropical, Goiânia, v. 38, n. 2, p. 109-117, 2008.

SANTOS, A. L.; MONNERAT, P. H.; CARVALHO, A. J. C. Estabelecimento de normas DRIS para o disgnóstico nutricional do coqueiro anão verde na região Norte Fluminense. Revista Brasileira de Fruticultura, Jaboticabal, v. 26, n. 2, p. 330-334, 2004.

SERRA, A.P. et al. Beaufils ranges to asses the cotton nutrient status in the southern region of Mato Grosso. Revista Brasileira de Ciência do Solo, Viçosa, v. 36, n. 1, p. 171-181, 2012.

SHAPIRO, S.S.; WILK, M.B. An analysis of variance test for normality (complete samples). Biometrica, London, v. 52, n. 3-4, p. 591-611, 1965.

SILVA, F. A. S; AZEVEDO, C. A. V. Principal components analysis in the software Assistat - Statistical Attendance. In: WOLD CONGRESS ON COMPUTERS IN AGRICULTURE, 7., 2009, Reno. Anais... Reno: American Sociey of Agricultural and Biological Engineers, 2009. 1 CD-ROM.

SILVA, D. J.; FARIA, C. M. B.; Amostragem foliar de coqueiros e distribuição de fertilizantes. 1. ed. Petrolina: Embrapa Semi Árido, 2001. 2 p. (Instruções Técnicas, 47).

SILVEIRA, C. P.; NACHTIGALL, G. R.; MONTEIRO, F. A.; Norms for the diagnosis and recommendation integrated system for signal grass. Scientia Agrícola, Piracicaba, v. 62, n. 6, p. 513-519, 2005.

SOBRAL, L. F.; NOGUEIRA, L. C. Influência de nitrogênio e potássio, via fertirrigação, em atributos do solo, níveis críticos foliares e produção do coqueiro anão. Revista Brasileira de Ciência do Solo, Viçosa, v. 32, n. 4, p. 1675-1682, 2008.

SOBRAL, F. L. Nutrição e adubação do coqueiro. In: FERREIRA, J. M. S.; WARWICK, D. R. N.; SIQUEIRA, L. A. (Ed.). A cultura do coqueiro no Brasil. Aracaju: Embrapa/SPI, 1998. v. 2, cap. 6, p. 129-157.
TEIXEIRA, L. A. J. et al. Adubação com NPK em coqueiro anão verde (Cocos nucifera L.) - Atributos químicos do solo e nutrição da planta. Revista Brasileira de Fruticultura, Jaboticabal, v. 27, n. 1, p. 115-119, 2005

WADT, P. G. S.; DIAS, J. R. M. Normas DRIS regionais e inter regionais na avaliação nutricional de café Conilon. Pesquisa Agropecuária Brasileira, Brasília, v. 47, n. 6, p. 822-830, 2012.

WAIREGI, L. W. I.; VAN ASTEN, P. J. A. Norms for multivariate diagnosis of nutrient imbalance in arabica and robusta coffee in the east african highlands. Experimental Agriculture, Cambridge, v. 48, n. 3, p. 448-460, 2012.

WALWORTH, J. L.; SUMNER, M. E. The diagnosis and recommendation integrated system (DRIS). Advance in Soil Science, Madison, v. 6, n. 1, p. 149$188,1987$. 